\title{
EL PLACER EN EL LIBRO X DE LA ÉTICA NICOMÁQUEA. II PARTE
}

\author{
Pleasure in book X of Nicomachean Ethics. Part II.
}

\author{
Andrés Solano-Fallas*
}

\begin{abstract}
RESUMEN
Esta segunda parte del artículo tiene por intención continuar con la dilucidación de la postura de Aristóteles en torno al tópico de qué es el placer, según el Libro X de la Ética Nicomáquea (EN). Esto se hará mediante la exposición de las características restantes que no se pudieron abarcar en la primera parte, por razones de espacio. Cabe señalar que la intención del artículo constituye un posicionamiento en contra de una tradición filosófica que estudia este libro junto con el Libro VI de la Ética Eudemia (EE), porque considera este último como parte de EN.
\end{abstract}

Palabras clave: Aristóteles, placer, características, Libro X de EN.

\section{ABSTRACT}

The purpose of this second half is to continue elucidating Aristotle's position on the topic of pleasure, according to Book X of Nicomachean Ethics (NE). This will be done by an exposition of the remaining characteristics that couldn't be studied in the first half, due to its length. It is worth noting that the approach followed in this article takes a position against a philosophical tradition that studies this book along with Book VI of Eudemian Ethics (EE), because considers the last one as part of NE.

Key Words: Aristotle, pleasure, characteristics, Book X of NE. 


\subsection{Sexta característica}

Visto en qué consiste la última característica, y en relación con la primera, la presente tiene que ver con la limitación. El placer es limitado porque la $\dot{\varepsilon} v \dot{\varepsilon} \rho \gamma \varepsilon ı$ no es continua, dado que "todas las $\dot{v} v \dot{\varepsilon} \rho \gamma \varepsilon ı$ ı humanas son incapaces de actuar constantemente $y$, en consecuencia, tampoco se produce placer, pues

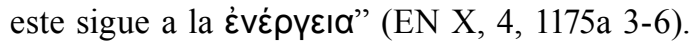

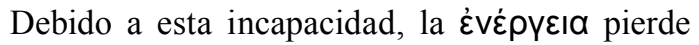
fuerza, dejando de ser la misma de cuando se correspondieron los elementos activos y pasivos. Dicha pérdida es la explicación de que "el placer se desvanezca" (EN X, 4, 1175a 10): al no haber $\dot{\varepsilon} v \dot{\varepsilon} \rho \gamma \varepsilon ı$, no hay placer. Lo mismo aplica, por consiguiente, al placer obtenido de la contemplación, con la salvedad de que es más prolongado, pues "somos más capaces de contemplar continuamente que de realizar

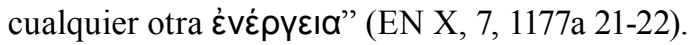

\subsection{Séptima característica}

Plantea que el placer es variado. No obstante, depende del sujeto que se trate, a saber, si es animal u hombre. Dado que hay diferencias en las variaciones de los placeres, la presente característica se dividirá. Pero antes de hacerlo, cabe señalar que no hay diferencia alguna en cómo surgen en los animales y en los hombres, debido a que el placer está relacionado con la

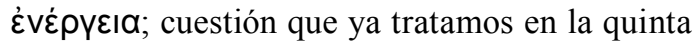
característica. Tanto en EN como en los tratados biológicos no se alude a una diferenciación en este respecto, por lo que asumimos que es de la misma manera.

\subsubsection{Variedad de los placeres en los animales}

En EN X, 5, 1176a 3-10, se considera que cada animal tiene su propio placer, ya que surge

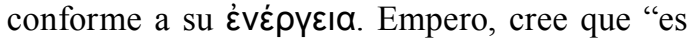
razonable pensar" que cada animal comparte los mismos placeres que les son propios a su especie. Por qué sea así, no da una explicación satisfactoria, solo indica que cada animal, entendido como especie, posee una función que le es propia. Con base en esta función propia, podría suponerse que los animales, al no ser seres intelectivos, no pueden contradecir lo que sus instintos les dictan, ya que se rigen de acuerdo con lo que les es propio. De esta manera, cada animal de determinada especie estaría actuando conforme a su naturaleza y compartiendo lo que de esta surja: los placeres.

$\mathrm{Si}$ tomamos mano de los tratados biológicos que dan cuenta del placer (1), nuestra suposición se vería confirmada. Brevemente, de acuerdo con el análisis descriptivo de cada una de las partes de los animales que realiza en De partibus animalium (PA), el Estagirita concluye que cada parte cumple una función según la anatomía del animal. Por ejemplo, en el caso de la lengua (II, 17, 660a 14 - 661a 34), apunta que "[t]odos los animales tienen deseo de alimento porque sienten la sensación de placer que procede del propio alimento (...). Pero el órgano con el que perciben el alimento no es igual en todos" (661a 5-10). De modo similar ocurre con los demás órganos, lo cual nos permite concluir y reforzar nuestra suposición: los animales de cada especie al compartir una fisiología y anatomía, están condicionados a tener los mismos tipos de placeres.

De ahí que le resulte a Aristóteles "razonable pensar" que cada animal comparte los placeres que les son propios a su especie. Sin embargo, lo anterior no quiere decir que cada animal de una especie lo comparta de la misma manera e intensidad. En Historia animalium (HA) V, 2, 539b 20-21, comenta particularmente del coito (de una determinada clase de animales según su clasificación (2)) lo siguiente: "En efecto, en los animales sanguíneos vivíparos provistos de patas, todos los machos poseen un órgano adecuado para el acto de la generación $y$, sin embargo, el acoplamiento no se hace en todos de la misma manera (...)".

A pesar de que esa clase de animales compartan dentro cada una de sus especies órganos adaptados para la procreación, en modo alguno supone que procreen de la misma manera; por lo que el placer que surja de la

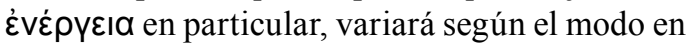
que se realice (además de los condicionamientos 
de la excelencia que comentamos en la quinta característica). Si bien Aristóteles se está refiriendo en HA concretamente al coito, no hay razón para negar que lo mismo suceda con otros placeres surgidos de otras દ̇vépүદıаı.

Como mencionamos, parte de nuestra suposición también consiste en afirmar que Aristóteles considera a los animales como seres no intelectivos. Este punto es reforzado, por un lado, en PA I, 1, 641b 9-10, cuando comenta que el alma de los animales no se refiere a todas las partes que constituyen el alma del hombre, pues "no hay que hablar sobre todas las partes del alma, pues no todas son naturaleza, sino alguna parte de ella, única o también varias." (3) En lo que respecta a la parte intelectiva del alma, previamente dice que no existe "el razonamiento en ningún otro". Por otro, en el capítulo 6 de De motu animalium (MA) 701a 5-6, indica que "el animal se mueve y avanza por deseo o elección, después de haber experimentado un cambio en la percepción o en la imaginación. (4)" Por tanto, se reafirma nuestra suposición de que los animales están determinados por sus instintos y a obtener los placeres que se desprenden de sus respectivas fisiologías y anatomías.

\subsubsection{Variedad de los placeres en los hombres}

A diferencia de cómo trató Aristóteles la diversidad de los placeres en el Libro VI de EE (VII de EN), en el presente no pretendió realizar una clasificación (por ejemplo, si son necesarios o no; o sin son excelentes y buenos o contrarios, o intermedios; o cómo son bienes y agradables). La variedad, por consiguiente, queda implícita cuando indica que la naturaleza humana no es simple, ya que "las mismas cosas agradan a unos y molestan a otros, y las hay molestas y odiosas para unos, y agradables y amables para otros" (EN X, 5, 1176a 10-12).

Así, contrario a lo que sucede en los animales, los hombres no comparten todos los placeres, a pesar de que compartan una fisiología y anatomía. La razón ya la mencionamos: la naturaleza humana es compleja. Esto nos muestra que en los animales la naturaleza es entendida en términos fisiológico-anatómicos, mientras que en los hombres la naturaleza no se reduce a esos términos, aunque sean parcialmente constitutivos e importantes en los grados de perfección, puesto que lo fisiológicoanatómico son los elementos activos.

Dada la falta de uniformidad en la naturaleza humana, a pesar de compartir los elementos activos, cabe preguntarse por qué es compleja. Pregunta importante, ya que es por esta falta de simpleza que los placeres no son los mismos para la especie humana. Para comprender qué es lo complejo de la naturaleza humana, debemos acudir a la breve antropologíapsicología que nos presenta en el capítulo 13 del Libro I de EN (1102a 26-1103a 3). Ahí nos dice que una parte del alma es irracional y la otra tiene razón, pero que dependiendo del tipo de hombre del cual se esté tratando, por ejemplo un continente o un incontinente, una parte del alma puede dominar sobre la otra, lo cual sin duda alguna afectará la escogencia del placer.

\subsection{Octava característica}

De acuerdo con la quinta y séptima característica, existen grados diversos de excelencia en los distintos placeres. Hasta este punto, pareciese no existir algún orden más concreto -y explicitado- de los placeres que la diferenciación entre placeres del pensamiento y de la contemplación y los placeres sensibles. En esta característica, Aristóteles presenta una suerte de cuadro general que clasifica los placeres con base en el grado de "pureza" (каӨаріо́тп). En EN X, 5, 1175b 36-1176a 3, tenemos lo siguiente: "La vista difiere del tacto en pureza, y el oído y el olfato del gusto; así los correspondientes placeres difieren del mismo modo, y de estos, los del pensamiento (סı́́voıa), y dentro de cada grupo, unos de otros".

Según lo anterior, se pueden distinguir, en términos generales, los placeres del "pensamiento" de los placeres sensibles; y dentro de cada uno de ellos, existen sus diferencias. Respecto de los placeres sensibles, tenemos que Aristóteles, en ese texto, solo ejemplifica que unos son más puros que otros, pero sin establecer un ordenamiento piramidal. 
A lo sumo tenemos que hay dos niveles: en un nivel alto se encuentran los placeres visuales, auditivos y olfatorios; y en un nivel menor, los tangibles y gustativos. Por qué los primeros tienen un grado de pureza mayor, no es algo que discuta en este libro.

En el capítulo 10 del Libro III de EN (1118a1 -1118b 8) encontramos que el Estagirita tiene igualmente en mayor estima a los placeres visuales, auditivos y olfatorios. Conviene aclarar que la estimación está centrada en el tema de la moderación e intemperancia, y no en el tema de la pureza. Dice que a nadie se le llama intemperante o moderado si disfruta de los placeres de la vista, del oído y del olfato. Solamente podría decirse que un hombre goza de esos placeres "como es debido, o con exceso o defecto" (EN III, 10, 1118 a 5-6). Con respecto a los placeres olfatorios, realiza una precisión: solo se le llama licencioso o moderado a aquellos hombres que "se deleitan con perfumes o [de los olores de] manjares. Pues los licenciosos se deleitan con estos, porque esto les recuerda el objeto de sus deseos" (EN III, 10, 1118a 6-8). Salvo estos olores, tampoco se le puede llamar a un hombre o moderado o licencioso.

Los placeres del tacto y del gusto, en cambio, "parecen serviles y bestiales" (EN III, 10, 1118a 25), porque son compartidos por los animales (5). Además, la moderación y la intemperancia tienen por objeto estos placeres, porque están propensos a excesos y defectos. ¿Qué es lo que los hacen propensos, a diferencia de los otros placeres que están "eximidos"? Tener un contacto más corporal y una vinculación mucho más directa con el objeto físico. Como señala Foucault (1990: 39), "no hay placer susceptible de akolasia si no hay tacto y contacto: contacto con la boca, la lengua y la garganta (para los placeres de la comida y de la bebida), contacto con otras partes del cuerpo (para el placer del sexo)." En lo concerniente al contacto con el cuerpo, Aristóteles realiza unas precisiones. Primero: "el gusto parece usarse poco o nada, porque lo propio del gusto es discernir los sabores" (EN III, 10, 1118a 27-28). Solo los intemperantes encuentran placeres en ello. El placer del gusto proviene cuando se tiene contacto "entero", "total" con la comida y la bebida, es decir, cuando no se busca discernir sabores. Segundo: existen ciertas excepciones en que la sobre medida en los placeres del tacto no es reprochable. Ello es cuando se trata de los más nobles, como por ejemplo "los que se producen en los gimnasios mediante las fricciones y el calor" (EN III, 10, 1118b 5-6). En estas precisiones puede notarse que el contacto corporal involucra un mayor contacto con el objeto físico, a diferencia de los placeres visuales, auditivos y olfatorios que, si bien sus placeres proceden de ciertos objetos físicos, no están vinculados del mismo modo que los placeres del tacto y del gusto.

En cuanto a los placeres del "pensamiento", tenemos que señalar que aquí el concepto "pensamiento" está siendo utilizado como una categoría que engloba otras $\dot{\varepsilon} v \dot{\varepsilon} \rho \gamma \varepsilon ı$ đI intelectuales, como la contemplación y lo que hemos entendido por pensamiento en la quinta característica, especialmente en la nota \# 26 de la primera parte de este trabajo. Recordemos que, como apuntamos en dicha nota, Aristóteles en otras ocasiones ha utilizado "pensamiento" de un modo general. Aquí, sin duda, parece tratarse de lo mismo, por lo que si no se tiene esto presente, podría crearse la ilusión de que en el Libro X la contemplación es parte del pensamiento. Hubiera sido más apropiado que el Estagirita utilizase otra categoría, como placeres

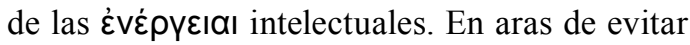
confusión entre "pensamiento" como categoría

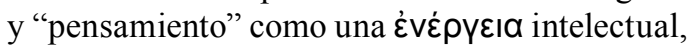
cambiaremos la primera por la que acabamos de

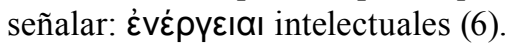

¿Cuáles son dichas દ̇vépyદıaı? Como se ve en el presente texto, Aristóteles no ofrece ejemplos. Por lo que hemos comentado en otras características (especialmente en la quinta), la contemplación y el pensamiento son sin duda alguna dos de ellas (7). Sus purezas parecen estar igualmente relacionadas al tema de la moderación y de la intemperancia. El Estagirita nos dice que los placeres que no afecten el cuerpo no son objetos de la moderación y de la intemperancia (cf. EN III, 10, 1117b 28-32). Si aplicamos la relación moderación/ 
intemperancia - pureza a los placeres de las

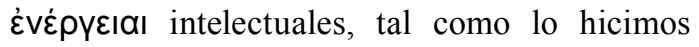
con los placeres sensibles, obtenemos que son más puros, debido a que no están ligados a lo corporal y a objetos físicos de la misma manera que los placeres del tacto y los gustativos; ni siquiera del mismo modo que los placeres visuales, auditivos y olfatorios.

Señalado lo anterior, ¿qué relación tiene la moderación y la intemperancia con la pureza? Aristóteles no la menciona, ni mucho menos dice en qué consiste la pureza. No obstante, puede proponerse que la pureza está ligada con el grado de moderación o intemperancia de los placeres. Como pudo haberse notado, parece que son más puros aquellos placeres que no son objeto de la moderación y de la temperancia

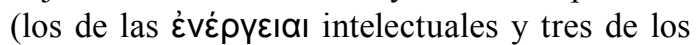
sensibles), mientras que aquellos que sí lo son, gozan de menor pureza. Consiguientemente, la pureza de los placeres parece consistir en que estos sean lo más independientes y alejados posibles del contacto corporal directo y de los objetos físicos.

Si lo anterior es correcto, en modo alguno, se sigue que Aristóteles propusiera con la pureza la eliminación o degradación de lo corporal. El Estagirita está consciente de que su hombre modelo -como los demás hombres- requiere, además de los bienes exteriores y anímicos, de los bienes corporales (EN I, 8, 1098b 13-14), y por ende, de ciertos placeres "más cercanos" a lo corporal, a saber, de los gustativos y del tacto.

Tampoco parece sugerir que la pureza determine o si quiera afecte la excelencia tal como la vimos en la quinta característica. La excelencia depende de la $\dot{\varepsilon} v \dot{\varepsilon} \rho \gamma \varepsilon ı$ : de la correspondencia entre el elemento pasivo y activo en ciertas condiciones. En ningún momento hizo entrar en juego la pureza; de ahí que afirmemos que la excelencia no depende de la pureza. Un placer del gusto, por ejemplo, puede ser más excelente que otro gustativo y por tanto más puro, y ambos siguen siendo menos puros que uno o varios placeres auditivos. Insistimos, debemos tener en cuenta que Aristóteles no discute dicha relación, puesto que no hay indicios textuales que lo muestren (8); solo nos apuntó que la pureza se da comparativamente de grupo a grupo, y dentro de cada grupo, sin incidir en la excelencia.

La pureza, por tanto, sería una suerte de criterio que los hombres (y sin duda alguna el hombre modelo), utilizarían para clasificar los placeres según su cercanía o lejanía de lo corporal y de los objetos físicos, sin que implique alguna determinación sobre la excelencia de los placeres. Asimismo, dicha clasificación les serviría para la escogencia del placer apropiado, según lo ameriten las circunstancias. Recordemos que el cuadro general que presenta esta característica no es producto del hombre modelo, sino que la pureza es parte de los placeres, pero que por sí mismo no basta, debido a que se requiere de una comparación para determinar cuál placer es más puro que otro en cierta circunstancia.

$$
* * *
$$

Esta característica parece haber sido una reformulación de la clasificación que el Estagirita realizó del placer en el Libro VI de EE (VII de EN). En tal libro se muestra un gran esfuerzo por dar cuenta de algunas características que son relativas a ciertos placeres, ya que la dificultad es grande, ardua, y hartamente compleja, y Aristóteles tuvo problemas en definir tales características, a pesar de que trató de ejemplificarlas para hacer la comprensión relativamente más fácil. Mientras en este libro hemos tenido el problema de que no es sistemático, a pesar de que en partes sea fragmentario, es conciso; en el Libro VI de EE (VII de EN), no solo no es sistemático, sino que en algunas partes es más caótico, puesto que algunas características presuponen un lector que conozca a qué se refiere con determinados términos. Además, no ofrece referencia a otra obra, o bien, ni siquiera los explica.

A nuestro criterio, esta característica no responde a una disputa, ni tampoco es parte de su postura (como las características quinta, sexta y sétima). Estamos convencidos de que es una auto-corrección metódica: en vez de tratar de trazar -digamos- una suerte de fenomenología de los placeres, ha preferido implementar una nueva clasificación y guía 
mucho más simple. Asimismo, nos resulta interesante que para ser una auto-corrección solo ocupe de unas cuantas líneas, ya que en esas pocas líneas prácticamente disuelve la tinta gastada en el Libro VI de EE (VII de EN) que no fue de manera alguna poca (9).

\section{Conclusión}

Recapitulando, el placer es completo, un todo en sí mismo; no es temporalmente mesurable en sí mismo, por lo que el tiempo no se puede percibir en él; sobreviene de la

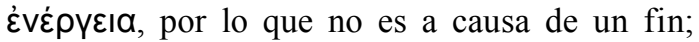
es inmaterial; perfecciona la $\dot{\varepsilon} v \dot{\varepsilon} \rho \gamma \varepsilon ı \alpha$ cuando

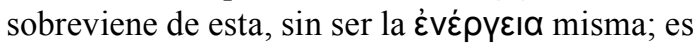
limitado, puesto que no es continuo; es variado (cuya variedad depende del sujeto en cuestión, a saber, si es hombre o animal); y, finalmente, posee grados de pureza que dependen según su cercanía o lejanía a lo corporal y a los objetos físicos. Las primeras cuatro características no fueron respuestas sistematizadas. A pesar de su brevedad, son directas. Su interés no era realizar, digamos, un tratado, sino en negar las posturas que él consideraba erróneas. Realizado esto, expone, sin la necesidad de refutar a otros, su postura en las características quinta, sexta y sétima; y se auto-corrige en la octava. En sentido negativo, se sigue que el placer no es ni está

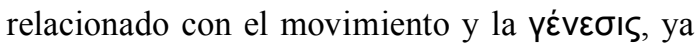
que estas son propias de las cosas materiales y divisibles. Los que afirmen que sea una de esas (o bien las dos) ni siquiera saben lo que están hablando cuando se refiere a uno (o los dos) de esos términos.

Para Aristóteles es importante clarificar lo que es el placer por la importantica que tiene en la vida de los hombres. Es un tema que no se puede pasar por alto, debido a que el placer perfecciona las $\dot{\varepsilon} v \dot{\varepsilon} \rho \gamma \varepsilon ı$ І que constituyen la vida, y por consiguiente, el vivir mismo "que todos desean. Es razonable, entonces, que aspiren también al placer, puesto que perfecciona la vida que cada uno ha escogido" (EN X, 4, 1175a 16-18) (10). Como Martos Montiel (19971998: 44) comenta, el Estagirita pasa "por alto la cuestión de si deseamos la vida por causa del placer o viceversa; simplemente, apunta entre ambos una relación de necesidad" ya que sin $\dot{\varepsilon} \vee \varepsilon \dot{\varepsilon} \rho \varepsilon^{\prime} \mid \alpha$ no hay placer, y el placer perfecciona

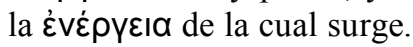

\section{Notas}

1. De los cinco tratados biológicos, solo tres hacen referencia al placer: Historia Animalium (HA), De Partibus Animalium (PA) y De Generatione Animalium (GA). No obstante, el Estagirita no abarca el tema del mismo modo en que EN y EE, ya que en estos tratados aparece disperso y siempre subordinado a otros intereses. Por ejemplo, en GA, cuando se refiere al placer, está hablando explícitamente del placer sexual en relación al coito y temas afines, tales como la producción de semen y catamenia. No teoriza sobre qué sea el placer. De manera similar ocurre en PA, en donde apunta que el placer depende de la fisiología y anatomía de cada animal, pero sin teorizar igualmente qué sea el placer. Claramente, su interés versa en torno a la descripción de las diversas fisiologías y anatomías animales y refutación de opiniones falsas. Procede del mismo en HA, aunque sean menos las referencias al placer.

2. En sentido estricto, Aristóteles no realizó una suerte de cuadro sinóptico donde se mostrase la clasificación antes de iniciar las investigaciones. No obstante, a partir de sus definiciones y descripciones de animales (y partes de los animales), se puede desprender uno sin forzar los textos; especialmente desde PA, seguido de HA. Para ver un cuadro sinóptico, referirse a Brun (1992: 114-115).

3. A nuestro juicio, la traducción castellana de esta cita parece algo confusa, por lo que decidimos brindar seguidamente la traducción inglesa (en Aristóteles, 1952): "[f] or it is not the whole soul that constitutes the animal nature, but only some part or parts of it."

4. El Estagirita no está reduciendo la sensación y la imaginación exclusivamente a la naturaleza animal. En el capítulo 6 de MA 700b 20, donde incluye brevemente al hombre, apunta que "tanto la imaginación como la sensación tienen el mismo lugar en la mente, pues todas son capaces de juzgar, y se distinguen por la diferencias citasa en otros lugares". Esos otros lugares en los cuales ha establecido la distinción están en Acerca del alma (deAn). Si bien hay diferencias notables en la división anímica entre EN y deAn -las 
cuales no trataremos acá, puesto que sobrepasa nuestros objetivos-, baste con hacer unos breves apuntamientos para comprender a qué se estaba refiriendo Aristóteles en MA.

Las distinciones se encuentran concretamente en el Libro II de deAn, en donde establece que "[e] n las plantas se da solamente la facultad nutritiva, mientras que en el resto de los vivientes se da no solo esta, sino también la sensitiva" (capítulo 3, 414a 31-414b 1). Mientras que a otros "les corresponde además la facultad discursiva y el intelecto: tal es el caso de los hombres y de cualquier otro ser semejante o más excelso, suponiendo que lo haya" (capítulo 3, 414b 15-19). En suma, a pesar de que la división anímica de deAn sea distinta a la formulada en EN I, 13, 1102a 26-1103a 3, los tres textos (deAn en relación con MA, y EN) nos muestran a Aristóteles excluyendo a los animales de ser intelectivos.

5. En EN III, 10, 1118a 16-23, señala que los animales no tienen placer en las sensaciones visuales, auditivas, y olfatorias. Si pareciese haber un placer, sería de manera accidental: "los perros no experimentan placer al oler las liebres, sino al comerlas; pero el olor puede producir la sensación; tampoco el león, con el mugido del buey, sino al devorarlo; pero se da cuenta de que está cerca por el mugido y, por ello, parece experimentar placer con él; igualmente, tampoco por ser $<<$ un ciervo o una cabra montés $>>$, sino porque tendrá comida."

Pero en las sensaciones del tacto y del gusto considera que los placeres sí tienen lugar.

6. Idea tomada de Broadie y Rowe (en Aristóteles, 2002b: 437). Los comentadores, sin dar alguna explicación o si quiera aludir al problema de "pensamiento" como categoría, cambian sin más la palabra "pensamiento" por "intellectual activities". Nos ha parecido oportuno tal cambio, por lo que lo hemos adoptado, aunque ofreciendo una explicación.

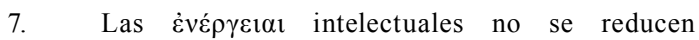
únicamente a estas dos. En otros libros ya ha mencionado otras. Sirva de ejemplo que en la Metafísica VI, 1, 1025b 25, señala el pensamiento práctico ( $\pi \rho \alpha \kappa \tau \iota \kappa o ́ \varsigma)$, el pensamiento productivo

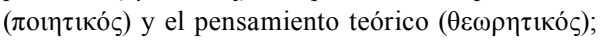
y en EE I, 6, 1217a 7, menciona el pensamiento

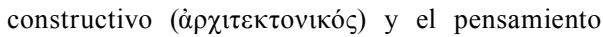

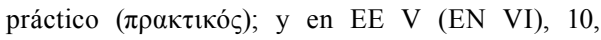

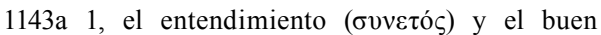

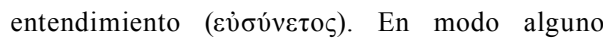
trataremos en qué consiste cada uno, ni mucho menos los ordenaremos según su grado de pureza. Baste su mera mención para darse una noción de que

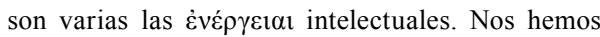
centrado en el pensamiento y en la contemplación, porque son estas dos las que Aristóteles menciona en el Libro X y las relaciona explícitamente con el placer.

8. Ha sido por esto por lo cual no hicimos la mínima mención de la pureza en la quinta característica, y la reservamos al final.

9. Para una mayor profundización y comparación, sírvase el lector de remitirse a nuestro artículo en dos partes en el cual se ha trabajado el placer en el Libro VI de EE (VII de EN) (Solano-Fallas, 2014b y 2015; próximos a publicarse); como también a nuestro otro artículo, en el que se realiza una comparación entre el Libro VI de EE y el presente que se estudia (Solano-Fallas, 2014a: 77-84).

10. No es nuestra intención destruir este último apuntamiento. Solo señalaremos que lo que dota de importancia al placer en la vida es la petición de principio de que "todos desean vivir". Aristóteles no se preocupa por fundamentar tal aseveración.

\section{Bibliografía}

Aristóteles. 1884. Aristotle's Eudemian Ethics (Edición de F. Susemihl). Leipzig: Teubner. Recuperado de http://www.perseus.tufts.edu Consulta octubre del 2011.

Para la Física, ver Aristóteles (1966).

Aristóteles. 1894. Aristotle's Ethica Nicomachea (Edición de J. Bywater). Oxford: Clarendon Press. Recuperado de http://www.perseus. tufts.edu Consulta octubre del 2011.

Aristóteles. 1924. Aristotle's Metaphysics (Edición de W. D. Ross). Oxford: Clarendon Press. Recuperado de http:// www.perseus.tufts.edu, y consultados constantemente entre los meses de mayo a octubre del 2011. 
Aristóteles. 1952. "History of Animals (Historia Animalium), Nicomachean Ethics (Ethica Nicomachea), Metaphysics (Metaphysica), On the Generation of Animals (De Generatione Animalium), On the Motion of Animals (De Motu Animalium), On the Parts of Animals (De Partibus Animalium), y Physics (Physica)". En: Benton, William (Ed.). The Works of Aristotle. (Dos volumenes). Chicago: Encyclopedia Britannica.

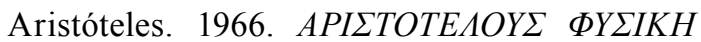

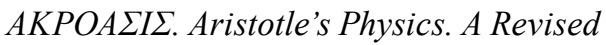
Text with Introduction and Commentary (por W.D. Ross). New York: Oxford University Press.

Aristóteles. 1978. Acerca del Alma. Madrid: Gredos.

Aristóteles. 1981. Gran Ética. Buenos Aires: Aguilar.

Aristóteles. 1992. Investigación de los animales. Madrid: Gredos.

Aristóteles. 1994a. Metafísica. Madrid: Gredos.

Aristóteles. 1994b. Reproducción de los animales. Madrid: Gredos.

Aristóteles. 1998. Acerca de la generación y la corrupción. Tratados breves de historia natural. Madrid: Gredos.

Aristóteles. 2000. Ética nicomáquea. Ética eudemia. Madrid: Gredos.

Aristóteles. 2000b. Partes de los animales. Marcha de los animales. Movimiento de los animales. Madrid: Gredos.

Aristóteles. 2002a. Física. Madrid: Gredos.
Aristóteles. 2002b. Nicomachean Ethics. Translation, Introduction and Commentary (por Sarah Broadie y Christopher Rowe). New York: Oxford University Press.

\section{Fuentes secundarias:}

Bravo, Francisco. 2009. As ambigüidades do prazer. Ensaio sobre o prazer na filosofia de Platão. São Paulo: Paulus.

Brun, Jean. 1992. Aristóteles y el Liceo. Barcelona: Paidós.

Fallas, Luis Alberto. "El placer en la filosofía griega y el mundo hebreo". En: Cárdenas, Luz Gloria, y Luis Alberto Fallas (2003). En diálogo con los griegos. Introducción a la filosofía antigua. Bogotá: Sao Pablo.

Foucault, Michel. 1990. Historia de la sexualidad. 2. El uso de los placeres. México: Siglo XXI.

González, Francisco. 1991. "Aristotle on pleasure and perfection". En: Phronesis. XXXVI /2: 141-159.

Hutchinson, D. S. "Ethics”. En: Barnes, Jonathan (Ed.) (1999). The Cambridge Companion to Aristotle. Cambridge: Cambridge University Press.

Kenny, Anthony. 1992. Aristotle on the Perfect Life. New York: Oxford University Press.

Kenny, Anthony. 1978. The Aristotelian Ethics. A Study of the Relationship Between the Eudemian and Nichomachean Ethics of Aristotle. New York: Oxford University Press.

Martos Montiel, Juan Francisco. 1997-1998. "El placer en las Éticas de Aristóteles". En: Excerpta Philologica 7-8: 33-47. 
Mesquita, Antonio Pedro. 2005. Introdução Geral. Volume I. Tomo I (Parte de Aristóteles. Obras Completas. Coordinada por Antonio Pedro Mesquita). Lisboa: Centro de Filosofia da Universidade de Lisboa - Imprensa Nacional - Casa da Moeda.

Pakaluk, Michael. 2005. Aristotle's Nicomachean Ethics: An introduction. Cambrigde: Cambrigde University Press.

Ramírez, Edgar Roy. 1986. "Tiempo y Movimiento en Aristóteles". En: Revista de Filosofía de la Universidad de de Costa Rica. XXIV (60): 177-180.

Reale, Giovanni. 1992. Introducción a Aristóteles. Barcelona: Herder.

Ross, W. D. 1957. Aristóteles. Buenos Aires: Editorial Sudamericana.

Solano-Fallas, Andrés. 2014a. "Comparación de las características del placer, según el Libro VI de la Ética Eudemia (Libro VII de la Ética Nicomáquea) y el Libro X de la Ética Nicomáquea". En: Revista de Filosofía de la Universidad de Costa Rica. LIII (135): 77-84. Enero-Abril.

Solano-Fallas, Andrés. 2014b. "El placer en el libro VI de la Ética eudemia (libro VII de la Ética nicomáquea). I parte". En: Revista de Filosofía de la Universidad de
Costa Rica. LIII (137): 77-89. SetiembreDiciembre.

Solano-Fallas, Andrés. 2015. "El placer en el libro VI de la Ética eudemia (libro VII de la Ética nicomáquea). II parte”. En: Revista de Filosofía de la Universidad de Costa Rica. LIV (138): 83-91. Enero-Abril.

Strohl, Matthew. 2008. An interpretation of Aristotle's Theory of Pleasure. Tesis de Doctorado. Universidad de Princeton.

Weiss, Roslyn. 1979. “Aristotle's Criticism of Eudoxian Hedonism". En: Classical Philology. Vol. 74 (3): 214-221.

\section{Materiales de consulta para el griego antiguo:}

Sebastián Yarza, Florencio I. 1999. Diccionario Griego-Español (Dos Tomos). Barcelona: Ramón Sopena.

Liddell, Henry George, y Robert Scott. 1940. An Greek-English Lexicon (Versión revisada y aumentada por Sir Henry Stuart Jones). Oxford: Clarendon Press. Recuperado de www.perseus.tufts.edu Consulta octubre del 2011.

Liddell, Henry George, y Robert Scott. 1889. An Intemediate Greek-English Lexicon. Oxford: Clarendon Press. Recuperado de www.perseus.tufts.edu Consulta octubre del 2011.

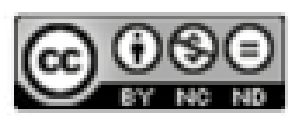

Este obra está bajo una licencia de Creative Commons Reconocimiento-NoComercial-SinObraDerivada 4.0 Internacional. 